\title{
Study on Gear Contact Stiffness and Backlash of Harmonic Drive Based on Fractal Theory
}

\section{Tao Zhang}

Beijing University of Technology

Zhifeng Liu

Jilin University

Congbin Yang ( $\nabla$ yangcongbin@bjut.edu.cn )

Beijing University of Technology

Yang Wang

Beijing Ctkm Harmonic Drive Co.,Ltd.

Qianqian Liu

Beijing Ctkm Harmonic Drive Co.,Ltd.

Original Article

Keywords: Harmonic drive, Contact stiffness, Gear backlash, Fractal theory

Posted Date: June 29th, 2021

DOI: https://doi.org/10.21203/rs.3.rs-652069/v1

License: (c) (i) This work is licensed under a Creative Commons Attribution 4.0 International License. Read Full License 


\section{Study on gear contact stiffness and backlash of harmonic}

\section{drive based on fractal theory}

Tao Zhang ${ }^{1}$, Zhifeng Liu ${ }^{2}$, Congbin Yang ${ }^{1 \#}$, Yang $\mathrm{Wang}^{3}$, Qianqian $\mathrm{Liu}^{3}$

1 Key Laboratory of advanced manufacturing technology, Beijing University of Technology, Beijing, 100124, P.R. China.

2 Key Laboratory of CNC Equipment Reliability, Ministry of Education, School of Mechanical and Aerospace Engineering, Jilin University, 130021, P.R. China.

3 Beijing Ctkm Harmonic Drive Co.,Ltd.,Beijing 101300,China

\# Corresponding Author / Email: yangcongbin@bjut.edu.cn.

Abstract: Contact stiffness and backlash model of harmonic reducer is related to robot's positioning accuracy and vibration characteristics. Harmonic reducer tooth pair height is typically less than $1 \mathrm{~mm}$. Thus, backlash and contact stiffness measurement and modeling are relatively complex. In this paper, contact stiffness and backlash model is proposed by establishing a relationship between fractal parameters and tooth contact load. Non-contact optical profiler and RMS method are combined to obtain fractal roughness parameters of real machined tooth surface. Finally, the effect of rough tooth surface and contact force fractal parameters on contact stiffness and gear backlash is studied. The results indicate that surface topography parameters and contact force have significant effects on contact stiffness and backlash. By increasing the fractal dimension, a decrease of gear backlash and contact stiffness is observed. However, the opposite is true for the fractal roughness parameter. Lastly, an increase in contact force improves the contact stiffness.

Keywords: Harmonic drive; Contact stiffness; Gear backlash; Fractal theory

\section{Introduction}

Harmonic drives (HD) are widely used in robot joints due to their high transmission ratios, excellent transmission accuracy, and relatively small sizes [1]. Core components of harmonic reducer are the circular spline (CS), flexible spline (FS), and wave generator [2]. When wave generator rotates, dozens of tooth pairs on CS and FS simultaneously mesh and drive. Contact stiffness and backlash tooth pair model is an indispensable part of dynamic research. Its accuracy is related to the positioning accuracy and vibration characteristics of harmonic drive [3]. Normally, gear meshing is regarded as a behavior of elastomeric bodies. Furthermore, gear contact is simplified to spring and damping. Therefore, harmonic drive tooth contact stiffness and backlash study represents an example of a dynamic problem.

Fractal contact model can be used to describe contact between machined surfaces. This model has been widely employed for contact modeling of engineering joints [4-6]. Mandelbrot [7] first proposed fractal concept, whose characteristics can be observed at the engineering surface. Furthermore, the author presented definitive overview of the 
origins of his ideas and their new applications. On this basis, Majumdar and Bhushan [8] further analyzed the contact theory of two-dimensional rough surface, more commonly known as the MB model. Yan and Komevpoulos [9] demonstrated fractal characteristics of an engineering surface. Furthermore, the authors described the variation of contact load and stress area with surface roughness parameters in a bolted connection, thus obtaining size independent contact model. Chen [10] investigated normal contact stiffness fractal model between two spheroidal joint surfaces considering friction. Liu et al. [11] introduced the tilt angle into the contact model of asperity with a rough surface. In addition, the authors established an inclination contact model (IA model) by employing fractal theory. According to the presented investigations, it can be observed that fractal theory is constantly being refined and upgraded when modeling rough surface contacts.

In addition to rough surfaces contact problem, fractal theory is also widely used in gear meshing investigations. Chen [12] employed fractal theory to express spur gear backlash. Dynamic performance and backlash were described using a fractal dimension parameter. Zhao [13] established an improved model for spur gear meshing characteristics analysis with consideration of fractal surface contact and friction. The author analyzed the effect of fractal parameters on mesh characteristics. Liu et al. [14, 15] proposed mathematical model of gear surface fractal parameters, finite element simulation load, and stiffness model. The contact pressure calculated by the finite element method is brought into the MB contact model to obtain a time-varying meshing stiffness model of involute gears and torsional stiffness model of cycloid gears. Based on the conducted literature survey, it is concluded that fractal theory can be effectively used to model gear contact.

Current harmonic reducer stiffness studies have mainly focused on torsional stiffness. Dhaouadi et al. [16] determined that harmonic gear hysteretic stiffness is mainly determined by friction behavior of multi tooth contact. Furthermore, the authors observed that flexible deformation of FS can only affect nonlinearity of the stiffness curve. Therefore, load torque is expressed as superposition of nonlinear stiffness and friction terms. Chen et al. [17] investigated distribution of meshing forces and loading backlashes under varied transmission torques. Ma et al. [18] studied causes of harmonic drive gear time-varying stiffness, number of meshing teeth, and meshing length under different torques. However, torsional stiffness model can only represent meshing performance of multiple teeth, not the meshing characteristics of a single tooth pair.

Contact between CS and FS tooth profiles of harmonic reducer can be equivalent to the contact model of two rough tooth surfaces. In this paper, a mathematic harmonic drive contact stiffness and backlash model based on fractal theory is proposed. In Section 1, relevant theoretical research of fractal theory employed in machined tooth surfaces is introduced. Furthermore, the reason why fractal theory is used in this paper 
is explained. In Section 2, gear contact stiffness and backlash model is established based on fractal parameters of machined tooth surface and contact theory. Then, graphics and tables are employed for case analysis in Section 3. Effect of the instantaneous load and fractal parameters on contact stiffness and backlash of surfaces is analyzed in Section 4. Lastly, conclusions are drawn in Section 5.

\section{Mathematical modeling}

Due to their high transmission accuracy and strong stability, harmonic reducers are widely used in industrial robot joint applications. Typical harmonic reducer consists of three parts: FS with a cylindrical cup, CS with an inner tooth rim, and wave generator with a flexible bearing. When harmonic reducer rotates, $30 \%$ of all teeth are simultaneously in contact. However, height of each contact tooth pair is less than $1 \mathrm{~mm}$, with most of them being approximately $0.5 \mathrm{~mm}$ high, as shown in Fig. 1 . This contributes to complexity of tooth profile and roughness parameters measurements.

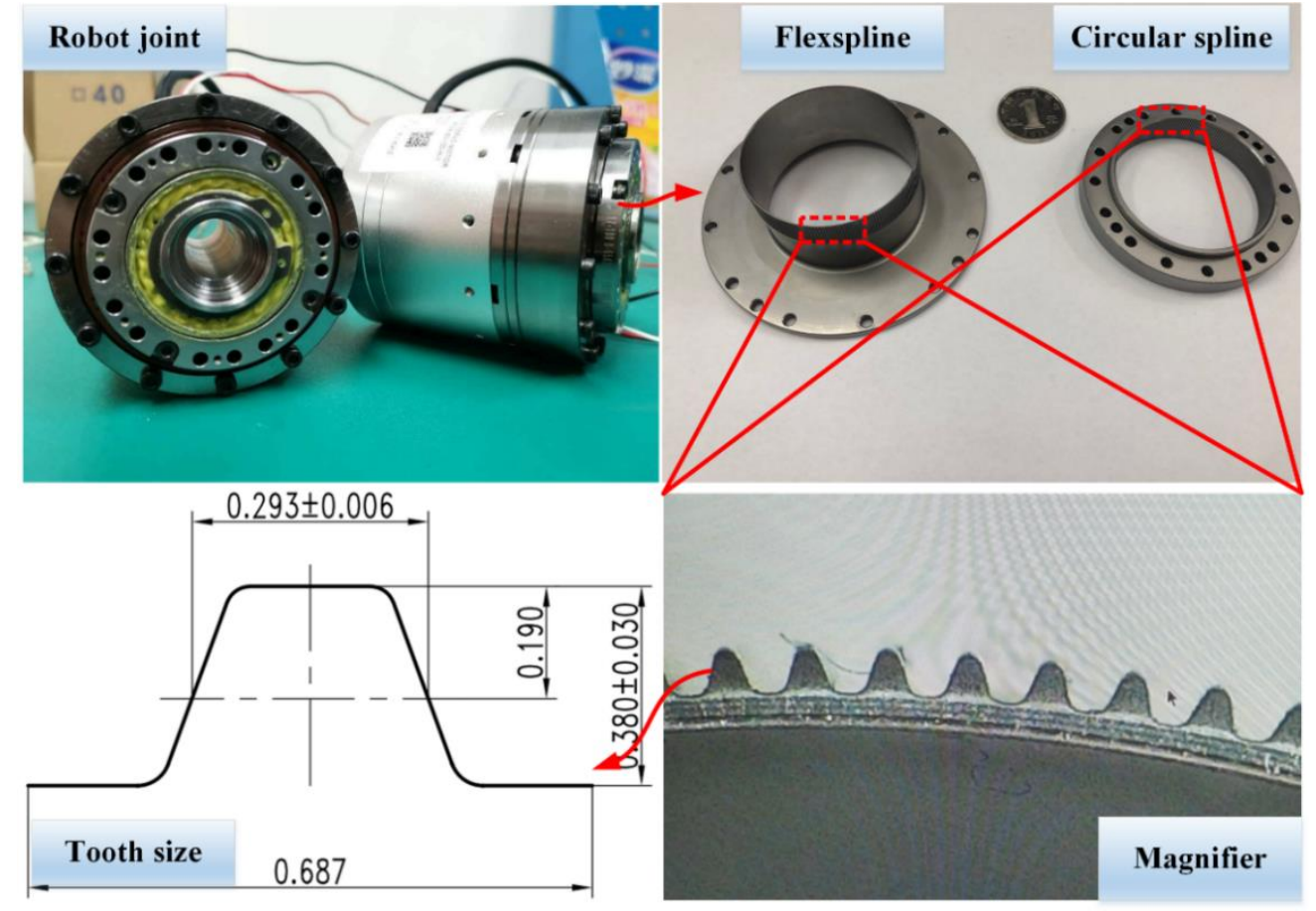

Fig. 1. Core parts and tooth profile of harmonic reducer

\subsection{Rough contact tooth surface modeling}

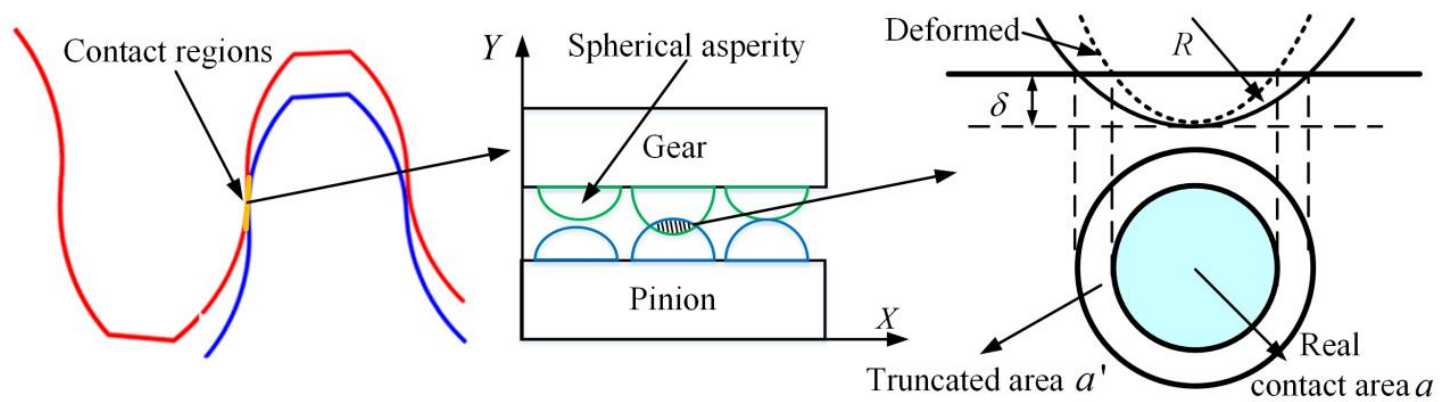

Fig. 2. Macroscopic and microscopic structure of tooth surface contact area 
Single tooth pair contact model of harmonic reducer is shown in Fig. 2. In fact, there are numerous asperities in smooth contact regions. These spherical asperities are actually rough surface micro features formed by gear machining. According to the M-B contact theory, two rough contact surfaces can be equivalent to one rough tooth surface in contact with another smooth tooth surface. In a similar manner, the contact model of two asperities can be simplified as one asperity contact with a plane. Actual contact area and deformation of each asperity depend on its size and the normal force acting on it.

Mathematical properties of machined tooth surfaces satisfy two dimensional Weierstrass-Mandelbrot (W-M) function [16]:

$$
z(x)=G^{(D-2)}(\ln \gamma)^{1 / 2}\left(2 r^{\prime}\right)^{3-D} \times\left\{\cos \phi_{1, n_{0}}-\cos \left(\frac{\pi x}{r^{\prime}}-\phi_{1, n_{0}}\right)\right\}
$$

Parameters employed in Eq. (1) are shown in Table 1.

Table 1. Parameters from Eq. (1)

\begin{tabular}{ll}
\hline Fractal parameters & meaning \\
\hline$D$ & $3 D$ fractal dimension $2<D<3$ \\
$G$ & fractal roughness \\
$x$ & abscissa of sampling \\
$\gamma$ & density of asperity $\gamma=1.5$ \\
$r^{\prime}$ & radius of single asperity truncated area \\
$\phi$ & random phase $0<\phi<2 \pi$ \\
$n_{0}$ & corresponding frequency index $n_{0}=\operatorname{int}\left[\log \left(L / 2 r^{\prime}\right) / \log \gamma\right]$ \\
$L$ & sampling length \\
\hline
\end{tabular}

For a single asperity, there are four important parameters: curvature radius $R$, normal deformation $\delta$, real contact area $a$, and truncated area $a^{\prime}$. Elastic or plastic deformation of an asperity can be distinguished by analyzing the relevant parameters. The corresponding relationship between geometric parameters of deformed asperity can be expressed as [23]:

$$
\begin{gathered}
R=\frac{a^{(\mathrm{D}-1) / 2}}{2^{(5-D)} \pi^{(\mathrm{D}-1) / 2} G^{(D-2)}(\ln \gamma)^{1 / 2}} \\
\quad \delta=2 G^{(D-2)}(\ln \gamma)^{\frac{1}{2}}\left(2 r^{\prime}\right)^{(3-D)}
\end{gathered}
$$

where the radius of truncated asperity can be described as $r^{\prime 2}=2 R \delta$.

The corresponding maximum elastic deformation $\delta_{c}$ and contact area $a_{c}^{\prime}$ are key indexes to evaluate whether the plastic deformation occurs or not, which can be given by:

$$
\delta_{c}=\left(\frac{\pi k H}{2 E}\right)^{2} R
$$




$$
a_{c}^{\prime}=\left[\frac{2^{11-2 D} G^{2(D-2)}(\ln \gamma) E^{2}}{\pi^{4-D}(k H)^{2}}\right]^{\frac{1}{D-2}}
$$

where parameter $E$ is the equivalent elastic modulus of two contact tooth pairs. $H$ is the hardness value of the softer tooth surface. Parameter $k$ can be obtained by an empirical formula related to the Poisson ratio $k=0.454+0.41 v$.

Three-dimensional distribution function model of asperity can then be defined as:

$$
n\left(a^{\prime}\right)=\frac{D-1}{2} \psi^{(3-D) / 2} a_{l}^{(\mathrm{D}-1) / 2} a^{\prime(-\mathrm{D}-1) / 2}
$$

where $a_{l}^{\prime}$ indicates the maximum cross-sectional area of the tooth surface asperity, and $\psi$ is related to the fractal dimension which can be obtained as:

$$
\frac{\psi^{(3-D) / 2}-\left(1+\psi^{(1-D) / 2}\right)^{-(3-D) /(D-1)}}{(3-D) /(D-1)}=1
$$

\subsection{Gear contact stiffness modeling}

Classical torsional vibration model of a gear pair is shown in Fig. 3. Time-varying stiffness $K_{m}$ is equivalent to a sum of contact stiffness of all tooth pairs along the meshing line. For harmonic drive, multiple tooth pairs are simultaneously meshing. Therefore, one meshing line cannot be employed. Hence, only the contact stiffness model of a single tooth pair is established in this paper.

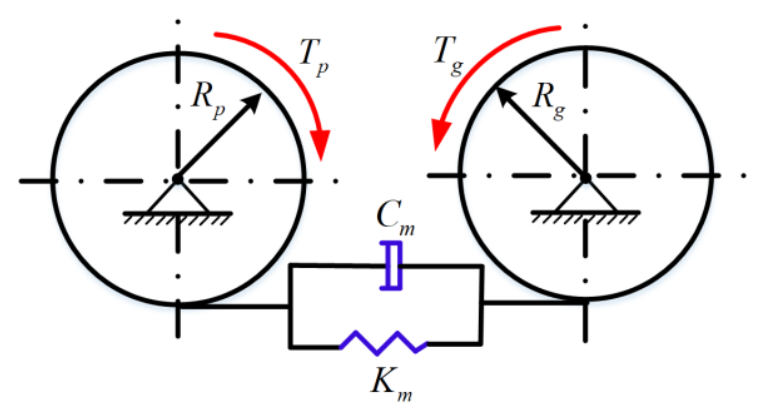

Fig. 3. Torsional vibration model of a gear-pair

During gear tooth meshing process, contact force is generated at the top of numerous asperities. According to different deformation stages [23], it can be divided into elastic contact force $f_{e}=4 / 3 E R^{1 / 2} \delta^{3 / 2}$ and plastic contact force $f_{p}=H a^{\prime}$. Total elastic normal load $F_{e}$ and plastic normal load $F_{p}$ can be obtained by integrating a distribution function of the asperity in Eq. (6).

$$
F_{e}=\left\{\begin{array}{l}
\frac{2^{(11-2 \mathrm{D}) / 2}}{3 \pi^{(4-\mathrm{D}) / 2}} \cdot \frac{D-1}{5-2 D}(\ln \gamma)^{1 / 2} G^{(\mathrm{D}-2)} E \psi^{(3-D) / 2} a_{l}^{(\mathrm{D}-1) / 2}\left(a_{l}^{\prime(5-2 D) / 2}-a_{c}^{\prime(5-2 D) / 2}\right)(D \neq 2.5) \\
2 \pi^{-3 / 4}(\ln \gamma)^{1 / 2} G^{1 / 2} E \psi^{1 / 4} a_{l}^{\prime 3 / 4} \ln \left(\frac{a_{l}^{\prime}}{a_{c}^{\prime}}\right) \quad(D=2.5)
\end{array}\right.
$$




$$
F_{p}=\frac{H(D-1) a_{l}^{(\mathrm{D}-1) / 2}}{3-D} \psi^{(3-D) / 2} a_{c}^{(3-D) / 2}
$$

Total normal load can be written as:

$$
F= \begin{cases}F_{e}+F_{p} & a_{c}{ }^{\prime}<a_{l}{ }^{\prime} \\ F_{p} & 0<a_{l}{ }^{\prime}<a_{c}{ }^{\prime}\end{cases}
$$

Micro asperities on the contact tooth surface produce contact force regardless of elastic or plastic deformation, but the contact stiffness only exists in the plastic deformation stage. Elastic contact stiffness $k_{e}$ is a reciprocal value of elastic contact pressure $f_{e}$ to normal deformation $\delta$ :

$$
k_{n e}=\frac{d f_{e} / d a^{\prime}}{d \delta / d a^{\prime}}=\frac{2 \sqrt{2} E(4-D)}{3 \sqrt{\pi}(3-D)} a^{1 / 2}
$$

In a similar manner, normal contact stiffness $K_{n e}$ is the integral of the contact stiffness of a single asperity $k_{e}$ and the distribution function $n\left(a^{\prime}\right)$ :

$$
K_{n e}=\int_{a_{1 c}^{\prime}}^{a_{l}^{\prime}} k_{n e} n\left(a^{\prime}\right) d a^{\prime}=\frac{2 \sqrt{2} E(4-D)(D-1)}{3 \sqrt{\pi}(3-D)(2-D)} \psi^{(3-D) / 2} a_{l}^{a^{(\mathrm{D}-1) / 2}}\left(a_{l}^{\left(^{(2-D) / 2}\right.}-a_{1 c}^{\prime(2-D) / 2}\right)
$$

According to a difference in maximum cross-sectional area $a^{\prime}$ of asperities under different load conditions, the normal contact stiffness $K_{m}$ of the tooth surface can be written as:

$$
K_{m}=\left\{\begin{array}{lc}
K_{n e} & a_{l}^{\prime}>a_{c}^{\prime} \\
0 & 0<a_{l}^{\prime}<a_{c}^{\prime}
\end{array}\right.
$$

\subsection{Gear backlash modeling}

Contact surface of machined gears produces clearance without considering profile modification. Therefore, gear backlash is defined by normal clearance distribution function, which has already been previously verified [12]. Function $f(x)$ is the backlash function, which is usually written according to Eq. (14).

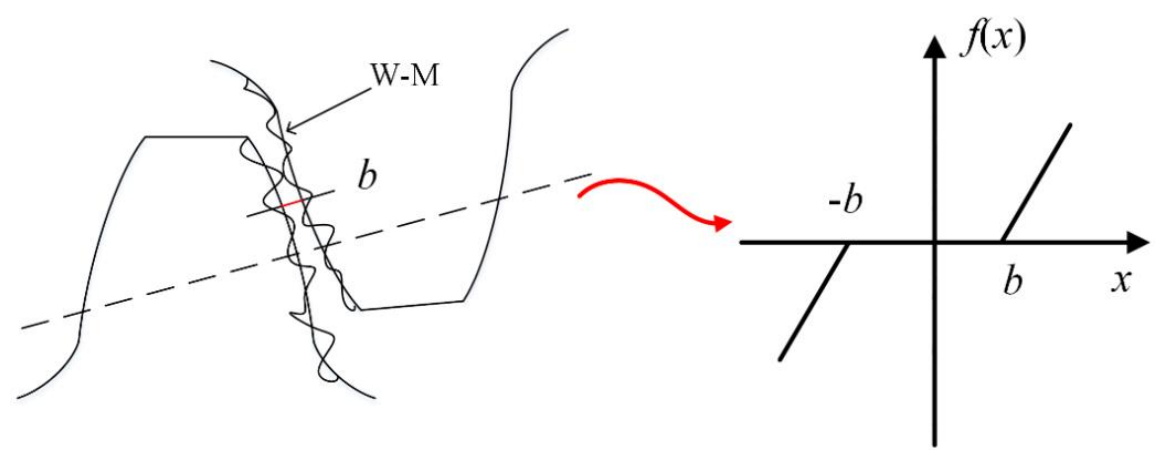

Fig. 4. Backlash function model of a gear-pair 


$$
f(x)= \begin{cases}x-b, & x>b \\ 0, & -b<x<b \\ x+b, & x<-b\end{cases}
$$

According to Chen et al. [12], backlash $b$ represented by W-M function significantly affects dynamic characteristics of gears. In this paper, W-M function is employed to describe the backlash function. Backlash $b$ described by three-dimensional Weierstrass-Mandelbrot (W-M) function can be written as:

$$
b=z(x)=G^{(D-2)}(\ln \gamma)^{1 / 2}\left(2 r^{\prime}\right)^{3-D} \times\left\{\cos \phi_{1, n_{0}}-\cos \left(\frac{\pi x}{r^{\prime}}-\phi_{1, n_{0}}\right)\right\}
$$

Parameters presented in Eq. (15) are already defined according to Eq. (1).

\subsection{Fractal roughness and fractal dimension parameter identification method}

According to Eqs. (1 - 15), fractal roughness parameter $G$ and fractal dimension $D$ have to be determined to obtain contact stiffness $K_{N}$ and backlash $b$. Fractal parameters of rough tooth surface can be calculated by surface data collected via non-contact optical profiler. The calculation method of fractal dimension $D$ and $G$ has been presented in many literatures. According to [21] and [22], the characteristic parameter of tooth surface obtained by roughness length (RMS) methods is more accurate. Thus, the experimental research in this paper is carried out based on this model.

(1) According to [22], the structure-function $S(\xi)$ in RMS method can be represented as:

$$
S(\xi)=<(z(x+\xi)-z(x))^{2}>
$$

where $<>$ represents the mean value, and $z(x)$ is the same as WM function in Eq.(1).

If Eq. (1) is combined with Eq. (16), it be expressed as:

$$
S(\xi)=C G_{s}^{2\left(D_{s}-1\right)} \xi^{\left(4-2 D_{s}\right)}
$$

Fractal roughness $G=<G_{s}>$,parameter $\mathrm{C}$ is equal to:

$$
C=\frac{\Gamma\left(2 D_{s}-3\right) \sin \left(\left(2 D_{s}-3\right) \pi / 2\right)}{\left(2-D_{s}\right)}
$$

where $\Gamma$ is the gamma equation of two-dimensional profile fractal dimension $D_{s}$. The relationship between a two-dimensional fractal dimension $D_{s}$ and three-dimensional fractal dimension $D$ is: $D=<D_{s}>+1$. According to Eq. (17), logarithm of $S(\xi)$ and $\xi$ can be used to obtain $D_{s}$ and $G_{s}$ via least-square fitting. 
Then, three-dimensional fractal dimensions $D$ and $G$ can be obtained from two-dimensional fractal dimensions $D_{s}$ and $D_{s}[4]$.

\section{Experimental setup}

Gear hobbing and shaping experiments are carried out to validate the proposed gear contact model and topography characteristics of machined tooth surface are analyzed. In this section, the experimental setup and tooth surface detection process is discussed in detail.YK3610 hobbing machine tool (made in Ning jiang, China) was used for gear profile hobbing tests, as shown in Fig. 5. 40CrNiMaA gear with 200 teeth, 0.25-module double circular arc profile, and $10.5 \mathrm{~mm}$ face width was machined. Non-contact optical profiler (ST-400) was employed to collect the parameters of FS tooth surface. In a similar manner, gear profile shaping machine tool (YGK512 made in Yi Chang, China) was utilized for hobbing tests. Prior to tooth profile scanning, CS inner ring gear was separated by wire cutting, as shown in Fig. 6. Details of the machined gear workpiece and non-contact optical profiler are listed in Tables 2 and 3, respectively.

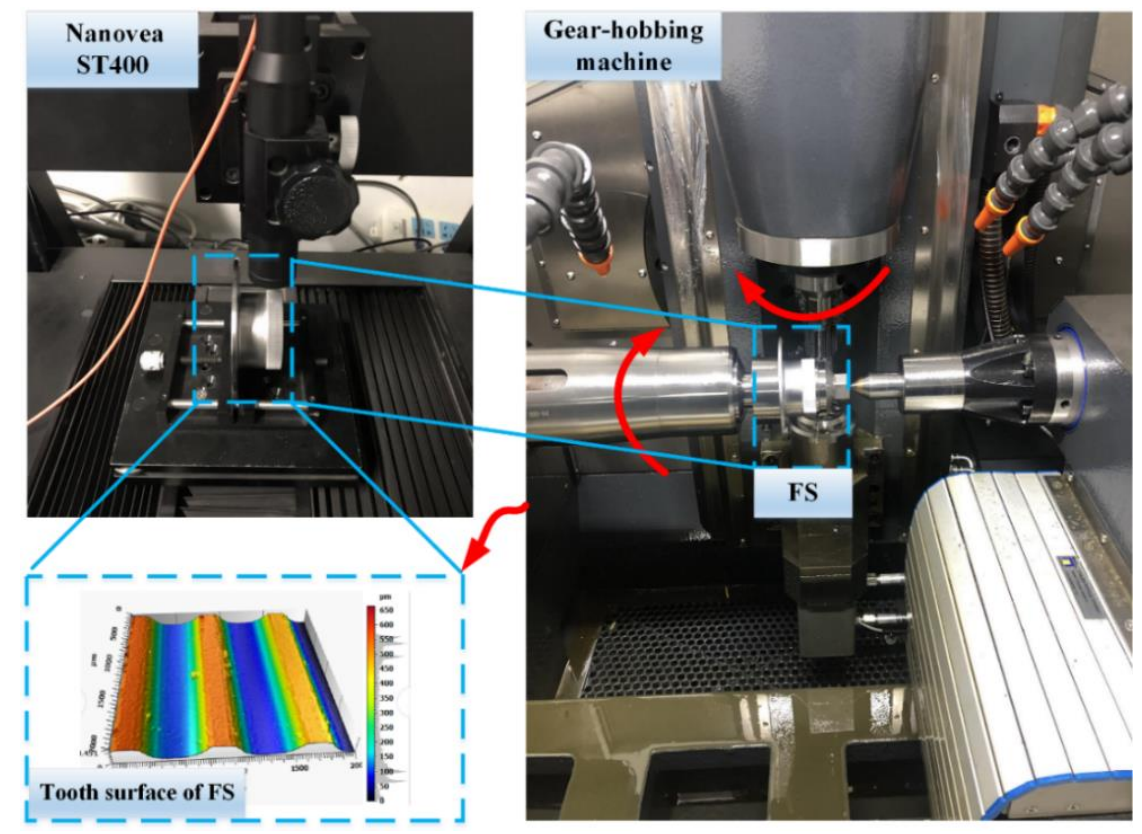

Fig. 5. FS teeth profile after hobbing

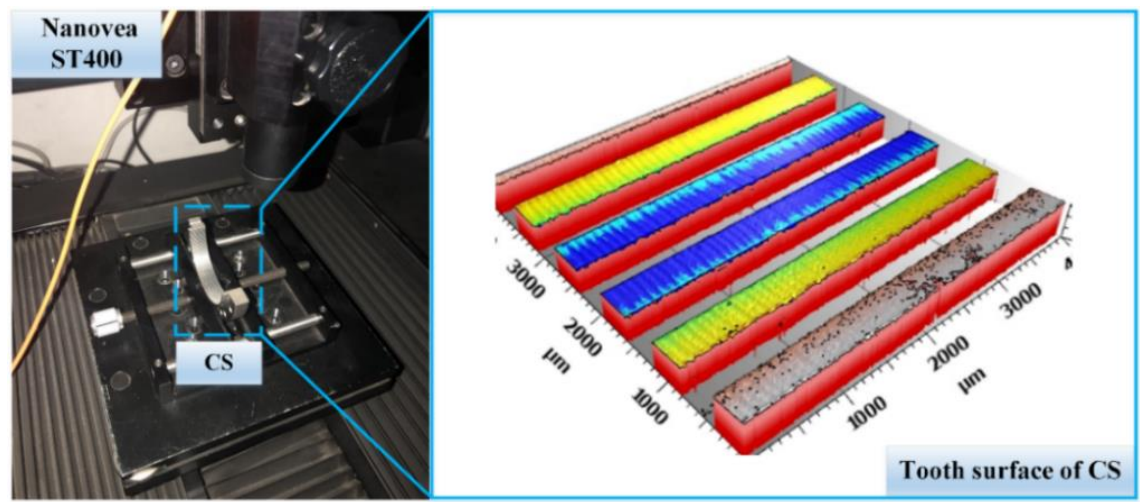

Fig. 6. CS teeth profile after gear shaping 
Table 2. Gear parameters

\begin{tabular}{|c|c|c|}
\hline Gear parameter & CS & FS \\
\hline Gear module & $0.25 \mathrm{~mm}$ & $0.25 \mathrm{~mm}$ \\
\hline Number of teeth & 202 & 200 \\
\hline Tooth shape & \multicolumn{2}{|c|}{ double circular arc } \\
\hline Pressure angle & \multicolumn{2}{|l|}{$10^{\circ}$} \\
\hline Face width & $10.5 \mathrm{~mm}$ & $10 \mathrm{~mm}$ \\
\hline Material of gear & 40CrNiMoA & $42 \mathrm{CrMo}$ \\
\hline \multicolumn{3}{|c|}{ Table 3. Measurement parameters } \\
\hline \multicolumn{2}{|c|}{ Measurement parameter } & Value \\
\hline \multicolumn{2}{|c|}{ Measurement equipment } & Nanovea ST400 \\
\hline \multicolumn{2}{|l|}{ Range } & $3500 \mathrm{um}$ \\
\hline \multicolumn{2}{|l|}{ Sampling interval } & $1 \mathrm{um}$ \\
\hline \multicolumn{2}{|l|}{ Resolving power } & $200 \mathrm{~nm}$ \\
\hline \multicolumn{2}{|l|}{ Measured area } & $4 \mathrm{~mm} \times 4 \mathrm{~mm}$ \\
\hline
\end{tabular}

According to optical instrument scanning results of tooth profile, tooth root arc, and tooth top arc, data are more complete than tooth profile data. Considering that the same tool under same working condition is used to process both tooth root and tooth profile, roughness parameter of root arc surface is employed to represent roughness parameters of the entire tooth profile, as shown in Fig.7. Region extracted from rough tooth surface data is hereinafter called "Interested tooth surface". Parameters of tooth surface roughness, including $S_{q}$ and $S_{a}$, can be directly extracted via ST400. RMS method curve should be calculated and drawn according to equation in Section 2.4.
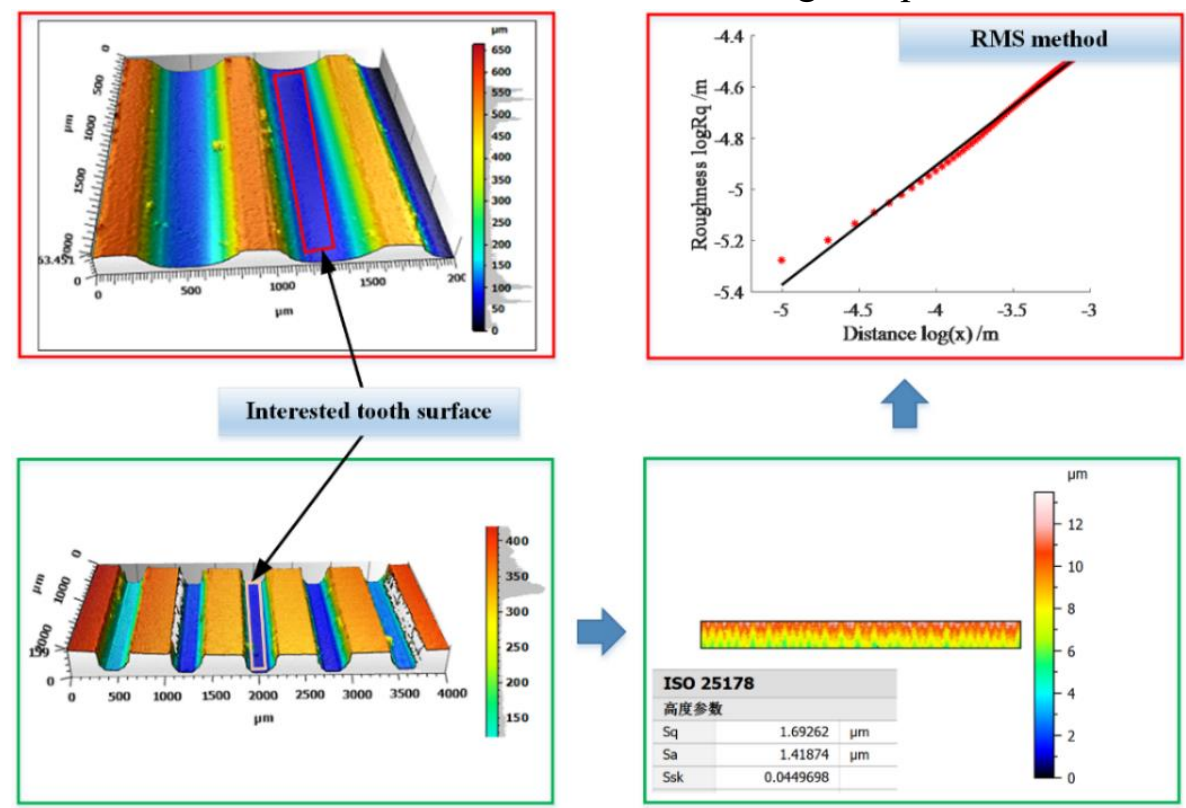

Fig. 7. Flowchart of RMS method

Final experimental results and test are listed in Table 4.

Table 4. Experimental and test results

\begin{tabular}{lll}
\hline Gear parameter & CS & FS \\
\hline
\end{tabular}




\begin{tabular}{lll}
\hline Fractal roughness $\boldsymbol{G}$ & $1.245 \times 10^{-10}$ & $1.8648 \times 10^{-10}$ \\
Fractal dimension $\boldsymbol{D}$ & 2.4272 & 2.643 \\
Equivalent roughness $\boldsymbol{G}$, & $1.55 \times 10^{-10}$ & \\
Equivalent dimension $\boldsymbol{D}$, & 2.5351 & \\
\hline
\end{tabular}

\section{Results and Discussion}

Tooth surface topography characteristics are discussed in this section. Effect of rough tooth surface fractal parameters and contact force on contact stiffness and gear backlash is studied.

\subsection{Effect of fractal parameters on backlash $b$}

Simulation of backlash $b$ with different fractal dimensions $D$ is shown in Fig. 8 . With an increase in fractal dimension, tooth surface becomes smoother. As shown in Table 4, average surface backlash value is used as the evaluation basis. In a similar manner, the smoother the tooth surface, the smaller the contact clearance. Therefore, fractal dimension of tooth surface should be approximately 2.5 to ensure micron level contact backlash.

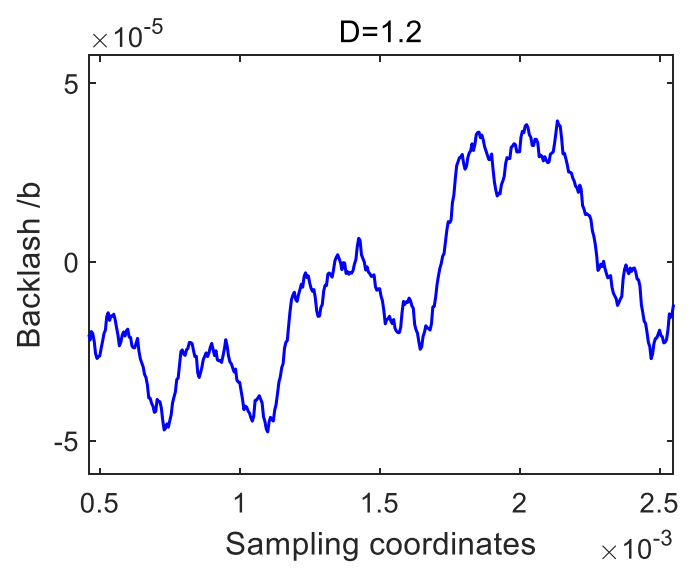

(a)

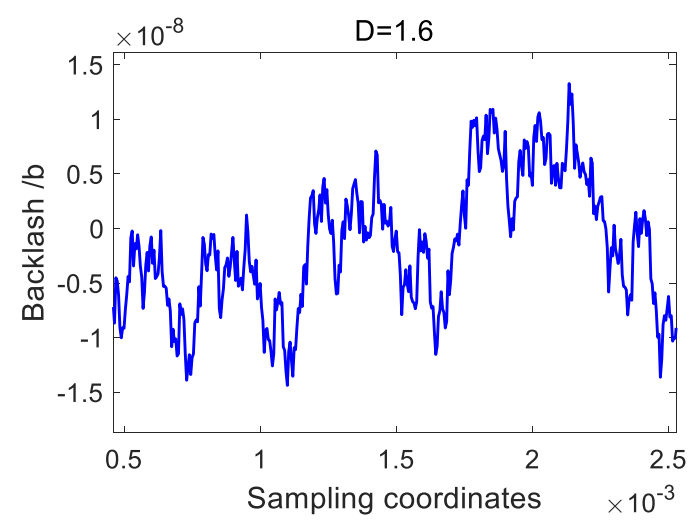

(c)

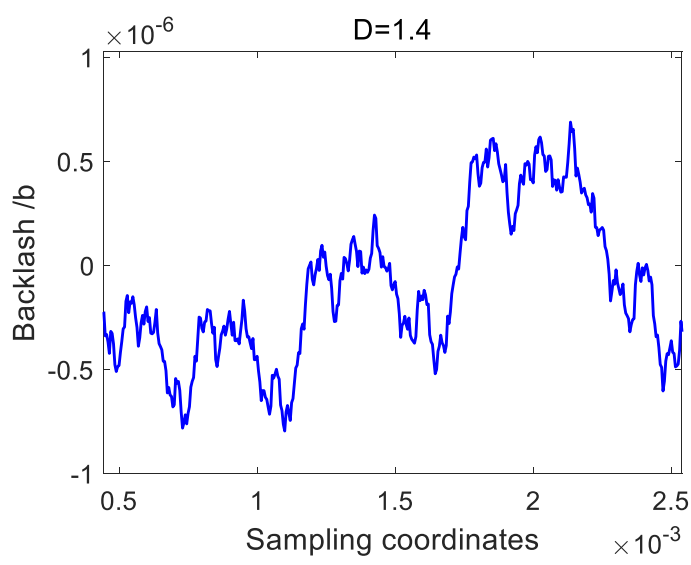

(b)

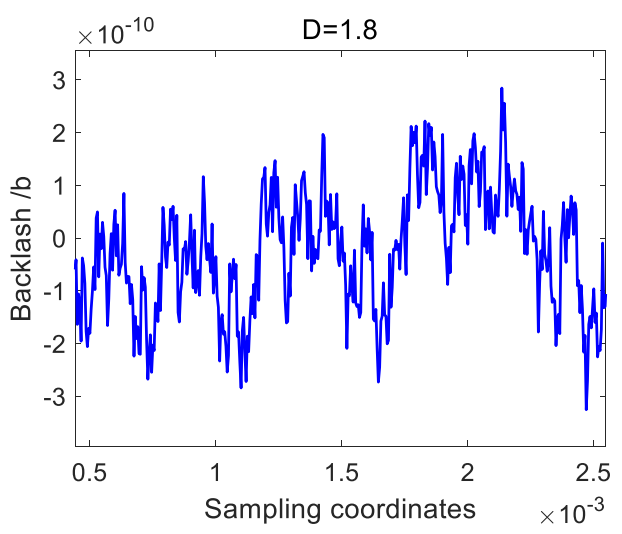

(d)

Fig. 8. Simulation of backlash (b) with different values of fractal dimensions (D)

In Fig. 9, backlash variation with different fractal roughness parameters $G$ is shown. When $G$ changes from $1 \mathrm{E}-9$ to $1 \mathrm{E}-15$, the tooth surface becomes smooth and backlash becomes uniform and stable. According to Table 4, $G$ should be less than $1 \mathrm{E}-13$ to ensure that the backlash reaches micron level. 


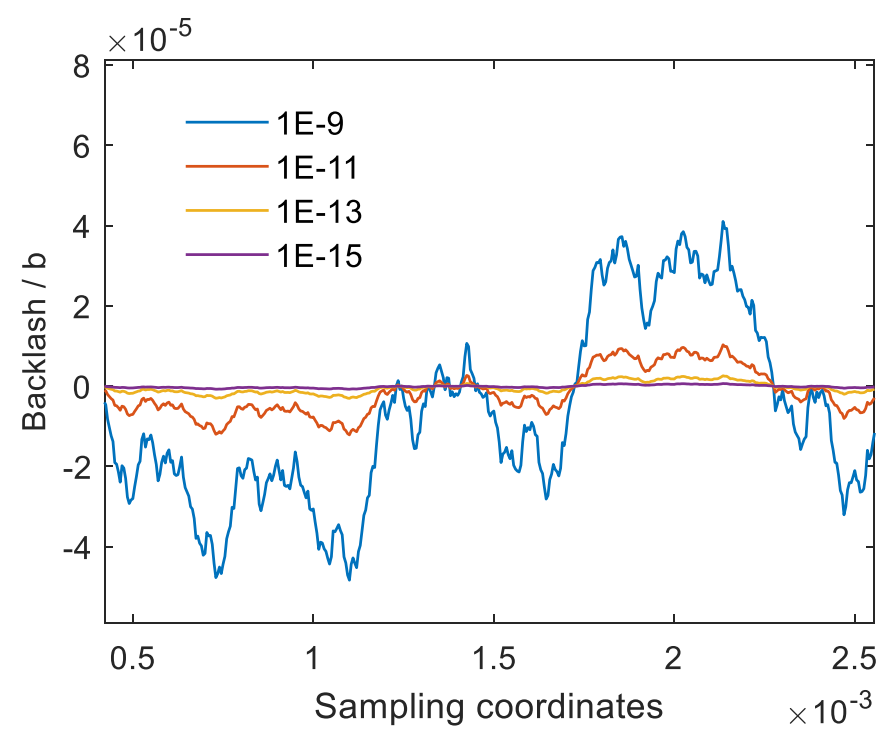

Fig. 9. Backlash simulation with different fractal roughness parameters Table 4. Average backlash for different values of $D$ and $G$.

\begin{tabular}{lll}
\hline $\boldsymbol{D}$ & $\boldsymbol{G}$ & $\bar{b}(\boldsymbol{\mu m})$ \\
\hline $\mathbf{2 . 2}$ & & 21.15 \\
$\mathbf{2 . 4}$ & $1 \mathrm{E}-12$ & 0.32 \\
$\mathbf{2 . 6}$ & & $5.13 \times 1 \mathrm{E}-3$ \\
$\mathbf{2 . 8}$ & & $9.42 \times 1 \mathrm{E}-5$ \\
& $1 \mathrm{E}-9$ & 20.52 \\
$\mathbf{2 . 3}$ & $1 \mathrm{E}-11$ & 5.15 \\
& $1 \mathrm{E}-13$ & 1.29 \\
& $1 \mathrm{E}-15$ & 0.33 \\
\hline
\end{tabular}

\subsection{Effect of fractal parameters on contact stiffness $K$}

Different fractal dimension and roughness parameters also affect the contact stiffness of the tooth surface. Contact pressure between CS and FS tooth of the harmonic reducer is assumed as $50 \mathrm{~N}$. Considering that the actual fractal dimension $D$ of machined tooth surface is between 2.3 and 2.6, stiffness variation law is discussed in this range. Variation of contact stiffness with tooth surface parameters is shown in Fig. 10.

According to Fig. 10(a), contact stiffness decreases with an increase in fractal dimension $D$. Combined with the tooth surface morphology demonstrated in Fig. 8, it is concluded that with an increase in micro convex body density, backlash and tooth surface contact stiffness are decreased. When tooth surface fractal roughness parameter $G$ changes according to Table 4, contact stiffness $K$ variation is shown in Fig. 10(b). An increase in fractal roughness parameter $G$ contributes to improving the contact stiffness. 


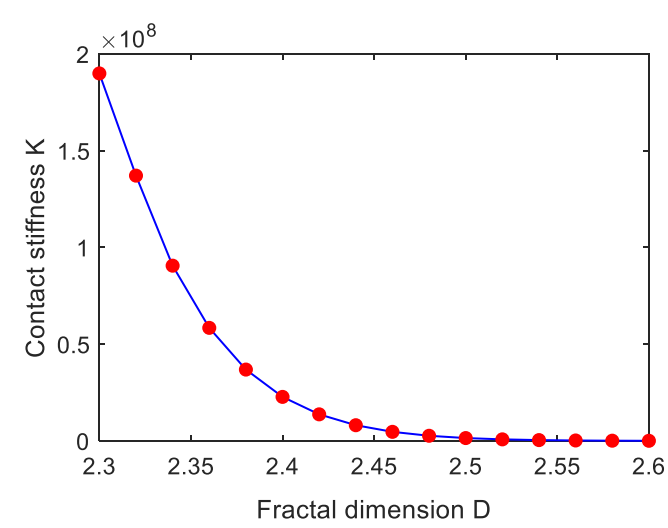

(a)

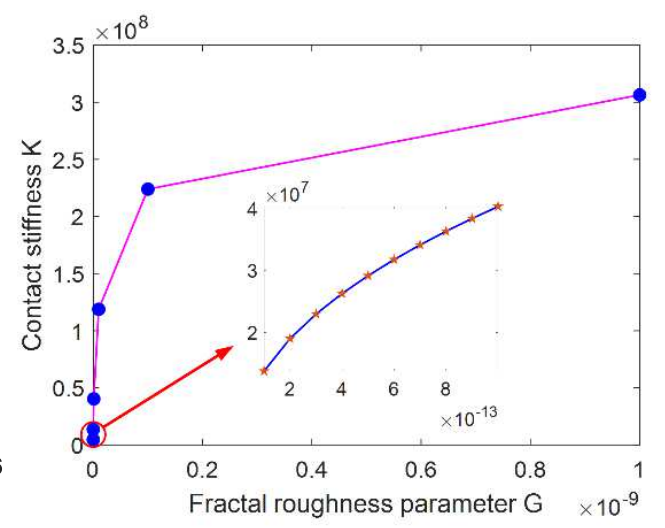

(b)

Fig. 10. Relationship between contact stiffness $K$ and fractal parameters

\subsection{Effect of contact force on contact stiffness $K$}

As previously mentioned, $30 \%$ of all teeth are simultaneously in contact in typical harmonic reducers. The contact position is considered to be on the indexing circle. Contact pressure of a single tooth pair under rated working load can be expressed as:

$$
F_{s}=\frac{T}{N d}
$$

where $T$ denotes the load torque, $N$ is the number of contact tooth pairs, and $d$ is diameter of the dividing circle.

Based on the provided data in Section 3 and Table 1, contact stiffness curves under different contact loads are investigated. Rated operating torque of this type of harmonic reducer is $52 \mathrm{Nm}$. Contact load under rated condition is $34.67 \mathrm{~N}$. Therefore, contact load range can be set between $10 \mathrm{~N}$ and $100 \mathrm{~N}$ to evaluate contact stiffness variation, as shown in Fig. 11.

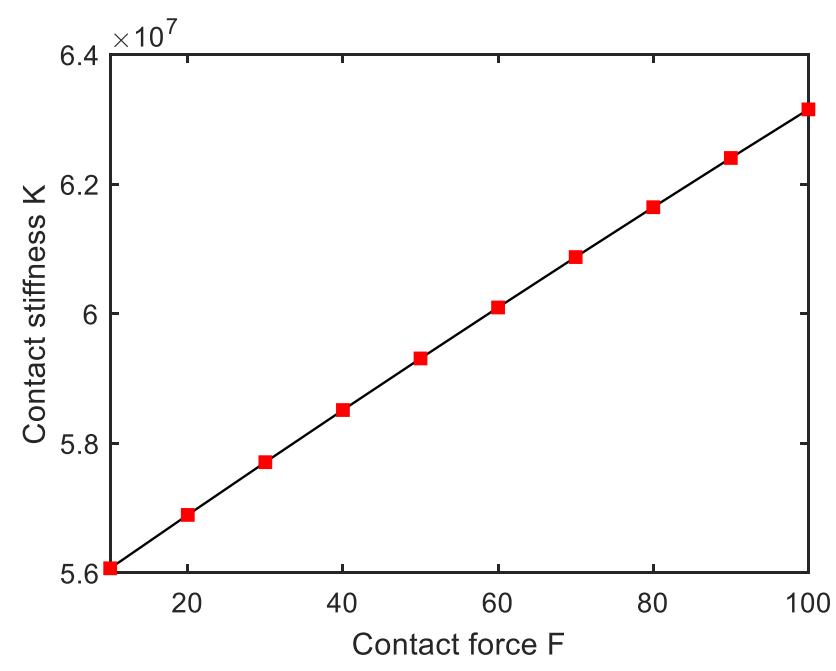

Fig. 11. Relationship between contact stiffness $\mathrm{K}$ and contact force $\mathrm{F}$

According to Fig. 11, contact stiffness increases linearly with contact pressure. Contact stiffness of harmonic reducer gear surface is $6 \times 10^{7}$ under rated condition. Experimentally obtained fractal parameters $D$ and $G$ are presented in in Table 3 . 


\section{Conclusions}

In this paper, mesh characteristics of harmonic gear contact tooth surfaces are theoretically and experimentally investigated. The following conclusions are drawn:

(1) Contact teeth surface of harmonic reducer can be described by M-B contact model. Furthermore, gear contact stiffness and backlash are functions related to fractal dimension $D$, fractal roughness parameter $G$, and contact load $F$.

(2) The results show that contact stiffness decreases with an increase in fractal dimension $D$. In addition, an increase in fractal roughness parameter $G$ and contact force $F$ contributes to improving the contact stiffness.

(3) Increasing fractal dimension $D$ and decreasing fractal roughness parameter $G$ can reduce the contact backlash.

In addition, this model is not only suitable for modeling the contact stiffness and backlash of harmonic drives, but also for identifying meshing parameters of gears with relatively small modules.

\section{Declarations}

Availability of data and materials: All data and materials are available.

Acknowledgments: The authors would like to thank the National Natural Science Foundation of China (51805012) and National Key R\&D Program of China (2020YFB2008200) for supporting the research.

Authors' contributions: Tao Zhang: Methodology, Writing-Original draft preparation. Zhifeng Liu: Conceptualization, Methodology, Supervision. Congbin Yang: Project administration, Writing- Reviewing and Editing. Yang Wang: Data curation. Qianqian Liu: Experimental verification.

Competing interests: There are no competing interests.

Funding: National Natural Science Foundation of China (51805012) and National Key R\&D Program of China (2020YFB2008200). 


\section{References}

[1]. Tjahjowidodo T, Al-Bender F, Van Brussel H. Theoretical modelling and experimental identification of nonlinear torsional behaviour in harmonic drives[J]. Mechatronics, 2013, 23(5):497-504

[2]. Ma DongHui, Wu JiaNing, Liu Tao, et al. Deformation analysis of the flexspline of harmonic drive gears considering the driving speed effect using laser sensors[J]. Science in China Series E: Technological Sciences. 2017,60,(8):1175-1187.

[3]. Huangfu Y, Chen K, Ma H, et al. Meshing and dynamic characteristics analysis of spalled gear systems: A theoretical and experimental study,[J]. Mechanical Systems and Signal Processing. 2020, 139: 106640.1-106640.21.

[4]. Zhifeng Liu, Kai Jiang, Xiangmin Dong, et al. A research method of bearing coefficient in fasteners based on the fractal and Florida theory[J]. Tribology International. 2020,152:106544..

[5]. Pawel Pawlus, Rafal Reizer, Michal Wieczorowski. A review of methods of random surface topography modeling[J]. Tribology International. 2020,152:106530..

[6]. Liu Jialan, Ma Chi, Wang Shilong, et al. Contact stiffness of spindle-tool holder based on fractal theory and multi-scale contact mechanics model[J]. Mechanical Systems and Signal Processing. 2019,119:363-379.

[7]. Mandelbrot, Benoit B. The Fractal Geometry of Nature. American Journal of Physics, 1983, 51(3):286.

[8]. Majumdar A, Bhushan B. Role of Fractal Geometry in Roughness Characterization and Contact Mechanics of Surfaces. Journal of Tribology, 1990, 112(2):205.

[9]. Yan W, Komvopoulos K. Contact analysis of elastic-plastic fractal surfaces. Journal of Applied Physics, 1998, 84(7):3617-3624.

[10]. Chen Qi, Xu Fan, Liu Peng, et al. Research on fractal model of normal contact stiffness between two spheroidal joint surfaces considering friction factor[J]. Tribology International. 2016,97:253-264..

[11]. Liu Zhifeng, Jiang Kai, Zhang Caixia, et al. A stiffness model of a joint surface with inclination based on fractal theory[J]. Precision Engineering-Journal of the International Societies for Precision Engineering and Nanotechnology. 2020,62:47-61.

[12]. Chen Q, Ma Y , Huang S, et al. Research on gears' dynamic performance influenced by gear backlash based on fractal theory[J]. Applied Surface science, 2014, 313:325-332.

[13]. Zhao Z , Han H , Wang P , et al. An improved model for meshing characteristics analysis of spur gears considering fractal surface contact and friction[J]. Mechanism and Machine Theory, 2021, 158:104219.

[14]. Liu Zhifeng, Zhang Tao, Zhao Yongsheng, et al. Time-varying stiffness model of spur gear considering the effect of surface morphology characteristics[J]. Proceedings of the Institution of Mechanical Engineers Part E-Journal of Process Mechanical Engineering. 2019,233,(2):242-253.

[15]. Liu Zhifeng, Zhang Tao, Wang Yida, et al. Experimental Studies on Torsional Stiffness of Cycloid Gear Based on Machining Parameters of Tooth Surfaces[J]. International Journal of Precision Engineering and Manufacturing. 2019,20,(6):1017-1025.

[16]. Dhaouadi R, Ghorbel F H, Gandhi P S. A new dynamic model of hysteresis in harmonic drives 
[J]. IEEE Transaction on Industrial Electronics, 2003, 50(6): 1165-1171.

[17]. Xiaoxia C , Pengpeng Y, Jingzhong X, et al. Research on Distribution of Loading Backlash and Meshing Force of Harmonic Drive[J]. journal of xi'an jiaotong university, 2018.

[18]. Ma J , Li C , Luo Y, et al. Simulation of meshing characteristics of harmonic reducer and experimental verification[J]. Advances in Mechanical Engineering, 2018, 10(3):168781401876749.

[19]. Zhuang Y, Li B T, Hong J, et al. A normal contact stiffness model of the interface[J]. Journal of Shanghai Jiaotong University, 2013, 47(2):180-186.

[20]. Kogut L, Etsion I A static friction model for elastic-plastic contacting rough surfaces. Journal of Tribology 2004, 126(1): 34-40

[21]. Zhang X, Xu Y, Jackson R L. An Analysis of Generated Fractal and Measured Rough Surfaces in Regards to Their Multi-scale Structure and Fractal Dimension. Tribology International, 2016, 105.

[22]. Feng F, Liu B, Zhang, X, Qian X. Roughness scaling extraction method for fractal dimension evaluation based on a single morphological image. Applied Surface Science, 2018; 458: 489494.

[23]. Zhao Yongsheng, Xu Jingjing, Cai Ligang, et al. Contact characteristic analysis of spindle-toolholder joint at high speeds based on the fractal model[J]. Proceedings of the Institution of Mechanical Engineers Part E-Journal of Process Mechanical Engineering. 2017,231,(5):1025-1036. 